\title{
Iridium-Catalyzed Asymmetric Allylic Amination of Allylic Alcohols Activated by Lewis Acids
}

\section{Supporting Information}

Yasuhiro Yamashita, Apsara Gopalarathnam and John F. Hartwig

Department of Chemistry, Yale University, P.O. Box 208107, New Haven, Connecticut 06520-8107 and Department of Chemistry, University of Illinois Urbana-Champaign, 600 South Mathews Avenue, Urbana, Illinois 61801 


\section{Table of Contents}

Page

1. General

2. General procedure for the enantioselective allylic amination

a. using a stoichiometric amount of niobium(V) ethoxide

3. General procedure for the enantioselective allylic amination

a. using a catalytic amount of triphenylborane 4

4. Spectral characterization of products $\quad 5$

5. Table S1. Asymmetric allylic substitutions of $\mathbf{1}$ with $\mathbf{2}$ in the presence of Ti alkoxides 13

6. Table S2. Screening of Lewis acid activator 14

7. Table S3. Screening of conditions with $\mathrm{Ti}\left(\mathrm{O}^{i} \mathrm{Pr}\right)_{4}, \mathrm{Nb}(\mathrm{OEt})_{5}$ and $\mathrm{Ta}(\mathrm{OEt})_{5}$ as activator 15

8. Figure S1. ${ }^{1} \mathrm{H}$ NMR of 4-chloro- $N$-(1-(4-methoxyphenyl)allyl)benzenamine 16

9. Figure S2. ${ }^{13} \mathrm{C}$ NMR of 4-chloro- $N$-(1-(4-methoxyphenyl)allyl)benzenamine 17

10. References 18 


\section{General:}

All manipulations were carried out under an inert atmosphere using a nitrogen-filled glovebox or standard Schlenk techniques. All glassware was oven dried immediately prior to use. THF was obtained as HPLC grade without inhibitors. Toluene was obtained as HPLC grade. tert-Butyl methyl ether (TBME), 1,2-dimethoxyethane (DME), and 1,4-dioxane were obtained as anhydrous grade packaged under nitrogen and used without further purification. THF and toluene were dried with a solvent purification system containing a $1 \mathrm{~m}$ column of activated alumina. All reagents were obtained from commercial sources and used without further purification unless otherwise noted. ${ }^{1} \mathrm{H}$ NMR spectra were obtained at $400 \mathrm{MHz}$ and recorded relative to solvent (chloroform: 7.26 ppm). ${ }^{13} \mathrm{C}$ NMR spectra were obtained at $125 \mathrm{MHz}$, and chemical shifts were recorded relative to the solvent (Chloroform: $77.0 \mathrm{ppm}$ ). As proof of purity of previously unknown compounds, elemental analysis or copies of spectra are provided. $[\operatorname{Ir}(\mathrm{COD}) \mathrm{Cl}]_{2}{ }^{1}$, $O, O$ '-(biphenyl-2,2'-diyl)- $N$-cyclododecyl- $N$-(1R)-1-phenylethylphosphoamidite $(\mathbf{5 a})^{2}$, and $O, O$ '- $(R)-\left(1,1\right.$ '-binaphthyl-2,2'-diyl)- $N, N$-bis-[(1R)-1-phenylethyl]phosphoamidite $\quad(\mathbf{5 b}){ }^{3}$ were synthesized according to published procedures. trans-Cinnamyl alcohol and trans-2-hexenyl alcohol were purchased from commercial sources. trans-Cinnamyl alcohol was recrystallized from ether and pentane before use, and trans-2-hexenyl alcohol was distilled before use. trans,trans-2,4-Hexadien-1-ol was purchased from commercial sources and used without further purification. trans-o-Methoxycinnamyl alcohol, trans-4-methylpet-2-en-1-ol, and trans-3-(2-furyl)-2-propen-1-ol were synthesized from corresponding aldehydes by simple reduction using $\mathrm{NaBH}_{4}$ in $\mathrm{MeOH}$. trans-p-Methoxycinnamyl alcohol was synthesized from trans-p-methoxycinnamic acid according to the literature method. ${ }^{4}$ Propylamine $\left(\mathrm{PrNH}_{2}\right)$ was purchased from commercial sources, distilled from calcium hydride, and stored under nitrogen. Powdered molecular sieves were purchased from Aldrich Chemical Co. and used after activation by heating at $250{ }^{\circ} \mathrm{C}$ for 1 day under vacuum. 
General procedure for the enantioselective allylic amination using a stoichiometric amount of niobium(V) ethoxide (Table 2). $[\operatorname{Ir}(\mathrm{COD}) \mathrm{Cl}]_{2}(0.0500 \mathrm{mmol})$ and phosphoamidite ligand $5(0.100 \mathrm{mmol})$ were placed in a $20 \mathrm{~mL}$ vial with a magnetic stir bar, and dissolved in THF $(0.50 \mathrm{~mL})$ and $\mathrm{PrNH}_{2}(0.50 \mathrm{~mL})$. The mixture was heated at $50{ }^{\circ} \mathrm{C}$ for $1 \mathrm{~h}$. After heating, all volatile materials were removed under reduced pressure. THF $(1.00 \mathrm{~mL})$ was then added to the vial to generate a stock solution of the iridium catalyst (0.100 M, $0.100 \mathrm{mmol}$ Ir catalyst in $1.00 \mathrm{~mL}$ of THF).

To a $4 \mathrm{~mL}$ vial were added allylic alcohol $1(1.00 \mathrm{mmol})$, powdered molecular sieves $4 \mathrm{~A}$ (50 mg), amine $2(1.50 \mathrm{mmol})$, THF $(0.30 \mathrm{~mL})$, a portion of the catalyst stock solution 0.20 $\mathrm{mL}(0.020 \mathrm{mmol})$ and $\mathrm{Nb}(\mathrm{OEt})_{5}(1.20 \mathrm{mmol})$. The mixture was stirred at $50{ }^{\circ} \mathrm{C}$ for $24 \mathrm{~h}$ unless otherwise noted. After cooling to room temperature, the reaction mixture was poured into a saturated sodium bicarbonate solution using methylene chloride. The mixture was stirred vigorously at room temperature for $1 \mathrm{~h}$. The mixture was filtered through Celite ${ }^{\circledR}$, and the solid remained was washed well with methylene chloride. After the organic phase was separated, the aqueous phase was extracted with methylene chloride twice. The organic phases were combined and washed with brine, and were dried over anhydrous sodium sulfate. After filtration and concentration in vacuo, the crude mixture was analyzed to determine the ratio of 3 to 4 by ${ }^{1} \mathrm{H}$ NMR spectroscopy using $\mathrm{CDCl}_{3}$ and one drop of $\mathrm{D}_{2} \mathrm{O}$. The addition of $\mathrm{D}_{2} \mathrm{O}$ removed a proton resonance that overlapped with the allylic signals of $\mathbf{3}$ and $\mathbf{4}$ used to measure this ratio. The crude mixture was purified with silica gel column chromatography (hexanes-ether) to afford the desired branched product 3 (and linear product $\mathbf{4}$ in the cases noted in Table 2).

General procedure for the enantioselective allylic amination using a catalytic amount of triphenylborane (Table 3). $\quad[\mathrm{Ir}(\mathrm{COD}) \mathrm{Cl}]_{2}(50.4 \mathrm{mg}, 0.0750 \mathrm{mmol})$ and $\mathbf{5 a}(75.2 \mathrm{mg}$, $0.150 \mathrm{mmol}$ ) were placed in a $20 \mathrm{~mL}$ vial with a magnetic stirrer bar and diluted with THF $(0.90 \mathrm{~mL})$ and $\mathrm{PrNH}_{2}(0.90 \mathrm{~mL})$. The mixture was heated at $50{ }^{\circ} \mathrm{C}$ for $1 \mathrm{~h}$. After heating, all volatile materials were removed under reduced pressure. 1,4-Dioxane $(1.50 \mathrm{~mL})$ was then added to the vial to generate a stock solution of the iridium catalyst $(0.1 \mathrm{M}, 0.150$ mmol Ir catalyst in $1.50 \mathrm{~mL}$ 1,4-dioxane). 
To a $20 \mathrm{~mL}$ vial were added the aniline (2) (1.00 mmol), the cinnamyl alcohol (1) (1.50 mmol), powdered molecular sieves $4 \AA$ (300 mg), 1,4-dioxane $(1.0 \mathrm{~mL}), 0.5 \mathrm{~mL}$ of the stock solution of the Ir catalyst $(0.050 \mathrm{mmol})$, and a solution of triphenylborane $(19.4 \mathrm{mg}$, $0.0801 \mathrm{mmol})$ in 1,4-dioxane $(0.50 \mathrm{~mL})$. The mixture was stirred at $50{ }^{\circ} \mathrm{C}$ for $24 \mathrm{~h}$. After cooling to room temperature, the reaction mixture was filtered, and poured into saturated sodium bicarbonate. The organic phase was separated, and the aqueous phase was extracted with ether. The ether phases were combined and washed with brine and dried over anhydrous $\mathrm{Na}_{2} \mathrm{SO}_{4}$. After filtration and concentration in vacuo, the crude mixture was purified by silica gel column chromatography (hexane:ether $=50: 1$ or hexanes: $\mathrm{CH}_{2} \mathrm{Cl}_{2}$ $=9: 1)$ to afford products 3 .

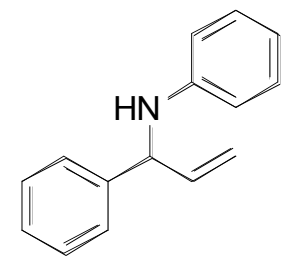

N-Phenyl-1-phenyl-2-propenylamine (Table 1): ${ }^{5} \mathrm{H}$ NMR (400 MHz, $\left.\mathrm{CDCl}_{3}\right)$ 7.40-7.32 (m, 4H), 7.30-7.24 (m, 1H), 7.17-7.11 (m, 2H), $6.70(\mathrm{t}$, $1 \mathrm{H}, J=7.3 \mathrm{~Hz}), 6.60$ (d, 2H, $J=7.9 \mathrm{~Hz}), 6.04(\mathrm{ddd}, 1 \mathrm{H}, J=17.1,10.2$, $5.9 \mathrm{~Hz}), 5.28(\mathrm{~d}, 1 \mathrm{H}, J=17.1 \mathrm{~Hz}), 5.22(\mathrm{~d}, 1 \mathrm{H}, J=10.2 \mathrm{~Hz}), 4.93(\mathrm{~d}, 1 \mathrm{H}$, $J=5.8 \mathrm{~Hz}), 4.1$ (br, $1 \mathrm{H}) ;{ }^{13} \mathrm{C} \mathrm{NMR}\left(125 \mathrm{MHz}, \mathrm{CDCl}_{3}\right)$ 147.2, 141.9, 139.1, 129.1, 128.7, 127.4, 127.1, 117.6, 116.0, 113.5, 60.8; EI-MS [m/z] $209\left(\mathrm{M}^{+}\right)$; HPLC (Daicel CHIRALCEL OJ-H; hexanes:2-propanol = 19:1; detection wavelength $=230 \mathrm{~nm}$; flow rate $=0.6 \mathrm{~mL} / \mathrm{min}$ ) $\operatorname{Tr}=30.8 \mathrm{~min}$ (major) and $35.7 \mathrm{~min}$ (minor).

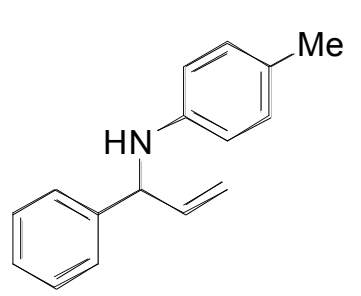

N-Tolyl-1-phenyl-2-propenylamine (Table 2, entry 1): ${ }^{5}{ }^{1} \mathrm{H}$ NMR (400 MHz, $\left.\mathrm{CDCl}_{3}\right)$ 7.39-7.31 (m, 4H), 7.29-7.22 (m, 1H), 6.97-6.92 (m, 2H), 6.55-6.50 (m, 2H), $6.03(\mathrm{ddd}, 1 \mathrm{H}, J=17.1,10.2,5.9 \mathrm{~Hz})$, $5.27(\mathrm{dt}, 1 \mathrm{H}, J=17.1,1.3 \mathrm{~Hz}), 5.21(\mathrm{dt}, 1 \mathrm{H}, J=10.2,1.2 \mathrm{~Hz}), 4.90$ $(\mathrm{d}, 1 \mathrm{H}, J=5.9 \mathrm{~Hz}), 3.92(\mathrm{br}, 1 \mathrm{H}), 2.21(\mathrm{~s}, 3 \mathrm{H}) ;{ }^{13} \mathrm{C} \mathrm{NMR}(125 \mathrm{MHz}$, $\left.\mathrm{CDCl}_{3}\right)$ 144.9, 142.1, 139.3, 129.6, 128.7, 127.4, 127.1, 126.8, 115.9, 113.7, 61.1, 20.3; EI-MS [m/z] $223\left(\mathrm{M}^{+}\right)$; HPLC (Daicel CHIRALCEL OD-H; hexanes:2-propanol = 99.7:0.03, detection wavelength $=230 \mathrm{~nm}$, flow rate $=0.5 \mathrm{~mL} / \mathrm{min}$ ) $\operatorname{Tr}=27.9 \mathrm{~min}$ (major) and $34.7 \mathrm{~min}$ (minor). 


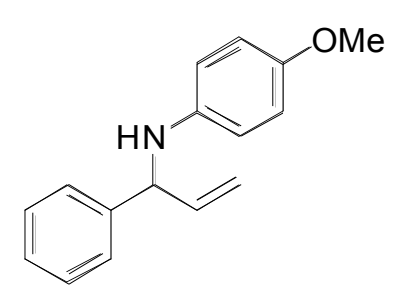

$N$-(p-Methoxyphenyl)-1-phenyl-2-propenylamine (Table 2, entry 2): ${ }^{1} \mathrm{H}$ NMR (400 MHz, $\left.\mathrm{CDCl}_{3}\right)$ 7.40-7.31 (m, 4H), 7.29-7.23 (m, 1H), 6.75-6.70 (m, 2H), 6.58-6.53 (m, 2H), 6.03 (ddd, $1 \mathrm{H}, J=17.1,10.2,6.0 \mathrm{~Hz}), 5.26(\mathrm{dt}, 1 \mathrm{H}, J=17.1,1.2 \mathrm{~Hz}$ ), $5.20(\mathrm{dt}, 1 \mathrm{H}, J=10.2,1.2 \mathrm{~Hz}), 4.85(\mathrm{~d}, 1 \mathrm{H}, J=6.0 \mathrm{~Hz}), 3.79$ (br, 1H), $3.71(\mathrm{~s}, 3 \mathrm{H}) ;{ }^{13} \mathrm{C}$ NMR (125 MHz, $\left.\mathrm{CDCl}_{3}\right)$ 152.2, 142.1, 141.4, 139.5, 128.7, 127.3, 127.1, 115.8, 114.9, 114.7, 61.8, 55.7; EI-MS [m/z] $239\left(\mathrm{M}^{+}\right)$; HPLC (Daicel CHIRALCEL OD-H; hexanes:2-propanol $=99.7: 0.03$, detection wavelength $=230 \mathrm{~nm}$, flow rate $=1.0$ $\mathrm{mL} / \mathrm{min}$ ) $\mathrm{Tr}=19.5 \mathrm{~min}$ (major) and $26.4 \mathrm{~min}$ (minor).

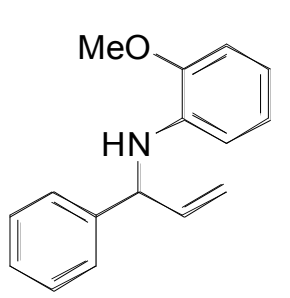

$\mathrm{N}$-(o-Methoxyphenyl)-1-phenyl-2-propenylamine (Table 2, entry 3) :

${ }^{1} \mathrm{H}$ NMR $\left(400 \mathrm{MHz}, \mathrm{CDCl}_{3}\right)$ 7.41-7.31 (m, 4H), 7.28-7.24 (m, 1H), 6.79-6.74 (m, 2H), 6.65 (ddd, $1 \mathrm{H}, J=8.3,7.1,1.5 \mathrm{~Hz}), 6.52-6.49(\mathrm{~m}$, $1 \mathrm{H}), 6.06(\mathrm{ddd}, 1 \mathrm{H}, J=17.1,10.2,5.9 \mathrm{~Hz}), 5.26(\mathrm{dt}, 1 \mathrm{H}, J=17.1,1.3$ $\mathrm{Hz}), 5.21(\mathrm{dt}, 1 \mathrm{H}, J=10.2,1.2 \mathrm{~Hz}), 4.92(\mathrm{~d}, 1 \mathrm{H}, J=5.8 \mathrm{~Hz}), 4.66(\mathrm{br}$, 1H), 3.86 (s, 3H); ${ }^{13} \mathrm{C}$ NMR (125 MHz, $\mathrm{CDCl}_{3}$ ) 146.8, 142.1, 139.3, 137.1, 128.7, 127.3, 127.1, 121.1, 116.7, 115.8, 111.2, 109.4, 60.7, 55.4; EI-MS [m/z] $239\left(\mathrm{M}^{+}\right) ;[\alpha]_{\mathrm{D}}^{20}=-4.3(\mathrm{c}$ 0.69, $\mathrm{CHCl}_{3}$ ); HPLC (Daicel CHIRALCEL OD-H; hexanes:2-propanol = 9:1, detection wavelength $=230 \mathrm{~nm}$, flow rate $=0.5 \mathrm{~mL} / \mathrm{min}$ ) $\mathrm{Tr}=10.5 \mathrm{~min}$ (minor) and $13.3 \mathrm{~min}$ (major); Anal. Calcd for C 80.30, H 7.16, N 5.85; Found: C 80.02, H 7.09, N 5.84.

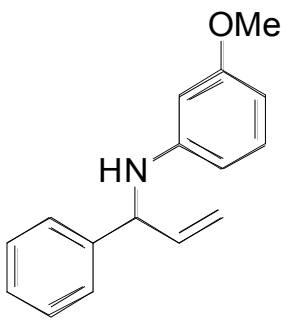

$\mathrm{N}$-(m-Methoxyphenyl)-1-phenyl-2-propenylamine (Table 2, entry 4): ${ }^{5} \mathrm{H}$ NMR $\left(400 \mathrm{MHz}, \mathrm{CDCl}_{3}\right)$ 7.39-7.31 (m, 4H), 7.29-7.24 (m, 1H), $7.04(\mathrm{t}, 1 \mathrm{H}, J=8.1 \mathrm{~Hz}), 6.26(\mathrm{dd}, 1 \mathrm{H}, J=8.1,2.3 \mathrm{~Hz}), 6.22(\mathrm{dd}, 1 \mathrm{H}, J=$ 8.0, $2.1 \mathrm{~Hz}), 6.16(\mathrm{t}, 1 \mathrm{H}, J=2.3 \mathrm{~Hz}), 6.03(\mathrm{ddd}, 1 \mathrm{H}, J=17.1,10.2,5.8$ $\mathrm{Hz}), 5.27(\mathrm{dt}, 1 \mathrm{H}, J=17.2,1.2 \mathrm{~Hz}), 5.22(\mathrm{dt}, 1 \mathrm{H}, J=10.2,1.2 \mathrm{~Hz}), 4.92$ (d, $1 \mathrm{H}, J=5.6 \mathrm{~Hz}), 4.06(\mathrm{br}, 1 \mathrm{H}), 3.72(\mathrm{~s}, 3 \mathrm{H}) ;{ }^{13} \mathrm{C} \mathrm{NMR}\left(125 \mathrm{MHz}, \mathrm{CDCl}_{3}\right)$ 160.6, 148.6, 141.8, 139.0, 129.8, 128.7, 127.4, 127.1, 116.1, 106.6, 102.8, 99.6, 60.8, 55.0; EI-MS [m/z] $239\left(\mathrm{M}^{+}\right)$; HPLC (Daicel CHIRALCEL OD-H; hexanes:2-propanol $=19: 1$, detection 
wavelength $=230 \mathrm{~nm}$, flow rate $=0.5 \mathrm{~mL} / \mathrm{min}$ ) $\mathrm{Tr}=29.3 \mathrm{~min}$ (minor) and $43.1 \mathrm{~min}$ (major).

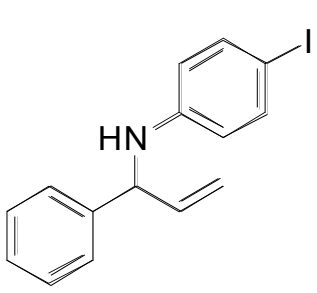

$N$-(p-Iodophenyl)-1-phenyl-2-propenylamine (Table 2 , entry 6): ${ }^{5}$ ${ }^{1} \mathrm{H}$ NMR (400 MHz, $\mathrm{CDCl}_{3}$ ) 7.39-7.25 (m, 7H), 6.40-6.34 (m, 2H), 6.01 (ddd, $1 \mathrm{H}, J=17.1,10.2,5.8 \mathrm{~Hz}), 5.25(\mathrm{dt}, 1 \mathrm{H}, J=17.1,1.2 \mathrm{~Hz})$, $5.23(\mathrm{dt}, 1 \mathrm{H}, J=10.2,1.2 \mathrm{~Hz}), 4.88(\mathrm{t}, 1 \mathrm{H}, J=4.7 \mathrm{~Hz}), 4.08(\mathrm{br}, 1 \mathrm{H})$; ${ }^{13} \mathrm{C}$ NMR $\left(125 \mathrm{MHz}, \mathrm{CDCl}_{3}\right)$ 146.7, 141.2, 138.5, 137.6, 128.8, 127.6, 127.1, 116.3, 115.8, 78.3, 60.6; EI-MS [m/z] $335\left(\mathrm{M}^{+}\right)$; HPLC (Daicel CHIRALCEL OD-H; hexanes:2-propanol $=99.7: 0.3$, detection wavelength $=230 \mathrm{~nm}$, flow rate $=0.5$ $\mathrm{mL} / \mathrm{min}$ ) $\operatorname{Tr}=40.0 \mathrm{~min}$ (major) and $57.1 \mathrm{~min}$ (minor).

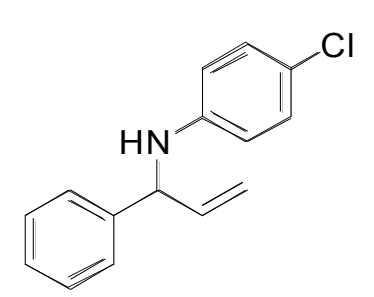

$N$-(p-Chlorophenyl)-1-phenyl-2-propenylamine (Table 2, entry 7): ${ }^{5}{ }^{1} \mathrm{H}$ NMR (400 MHz, $\left.\mathrm{CDCl}_{3}\right)$ 7.37-7.26 (m, 5H), 7.09-7.04 (m, 2H), 6.53-6.47 (m, 2H), $6.02(\mathrm{ddd}, 1 \mathrm{H}, J=17.1,10.2,5.8 \mathrm{~Hz}), 5.26$ $(\mathrm{dt}, 1 \mathrm{H}, J=17.1,1.1 \mathrm{~Hz}), 5.23(\mathrm{dt}, 1 \mathrm{H}, J=10.2,1.1 \mathrm{~Hz}), 4.88(\mathrm{~d}$, $1 \mathrm{H}, J=5.8 \mathrm{~Hz}), 4.05(\mathrm{br}, 1 \mathrm{H}) ;{ }^{13} \mathrm{C} \mathrm{NMR}\left(125 \mathrm{MHz}, \mathrm{CDCl}_{3}\right) 145.7$, 141.4, 138.6, 128.9, 128.8, 127.6, 127.1, 122.2, 116.3, 114.6, 60.9; EI-MS [m/z] $243\left(\mathrm{M}^{+}\right)$; HPLC (Daicel CHIRALCEL OD-H; hexanes:2-propanol = 99.7:0.3, detection wavelength $=230 \mathrm{~nm}$, flow rate $=0.5 \mathrm{~mL} / \mathrm{min}$ ) $\mathrm{Tr}=32.5 \mathrm{~min}$ (major) and $50.3 \mathrm{~min}$ (minor).

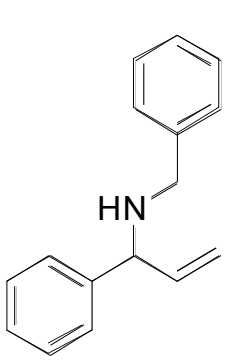

$N$-benzyl-1-phenyl-2-propenylamine (Table 2, entry 8): ${ }^{1} \mathrm{H}$ NMR (400 $\left.\mathrm{MHz}, \mathrm{CDCl}_{3}\right)$ 7.39-7.21 (m, 10H), $5.95(\mathrm{ddd}, 1 \mathrm{H}, J=17.1,10.2,7.2 \mathrm{~Hz})$, $5.23(\mathrm{dt}, 1 \mathrm{H}, J=17.1,1.2 \mathrm{~Hz}), 5.12(\mathrm{~d}, 1 \mathrm{H}, J=10.2 \mathrm{~Hz}), 4.22(\mathrm{~d}, 1 \mathrm{H}, J=$ $7.2 \mathrm{~Hz}), 3.74(\mathrm{~d}, 1 \mathrm{H}, J=13.3 \mathrm{~Hz}), 3.72(\mathrm{~d}, 1 \mathrm{H}, J=13.3 \mathrm{~Hz}), 1.63$ (br, 1H); ${ }^{13} \mathrm{C}$ NMR (125 MHz, $\left.\mathrm{CDCl}_{3}\right)$ 142.8, 141.0, 140.5, 128.5, 128.3, 128.1, 127.3, 127.2, 126.9, 115.1, 65.1, 51.3; EI-MS [m/z] $222\left(\mathrm{M}^{+}-1\right), 223\left(\mathrm{M}^{+}\right)$; HPLC (Daicel CHIRALCEL OD-H; hexanes:2-propanol = 99.7:0.3, detection wavelength $=230 \mathrm{~nm}$, flow rate $=0.5 \mathrm{~mL} / \mathrm{min}$ ) $\mathrm{Tr}=15.6 \mathrm{~min}$ (major) and $19.4 \mathrm{~min}$ (minor). 


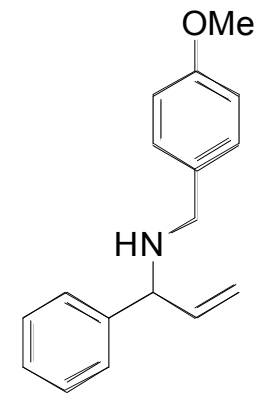

$N$-(p-Methoxyphenylmethyl)-1-phenyl-2-propenylamine (Table $\quad 2$, entry 9): ${ }^{6}{ }^{1} \mathrm{H}$ NMR (400 MHz, $\left.\mathrm{CDCl}_{3}\right)$ 7.38-7.31 (m, 4H), 7.28-7.21 (m, $3 \mathrm{H}), 6.87-6.83(\mathrm{~m}, 2 \mathrm{H}), 5.94(\mathrm{ddd}, 1 \mathrm{H}, J=17.1,10.2,7.2 \mathrm{~Hz}), 5.22$ (d, 1H, $J=17.1 \mathrm{~Hz}), 5.11(\mathrm{~d}, 1 \mathrm{H}, J=10.2 \mathrm{~Hz}), 4.21(\mathrm{~d}, 1 \mathrm{H}, J=7.2 \mathrm{~Hz}), 3.80(\mathrm{~s}$, $3 \mathrm{H}), 3.67(\mathrm{~d}, 1 \mathrm{H}, J=13.1 \mathrm{~Hz}), 3.65(\mathrm{~d}, 1 \mathrm{H}, J=13.1 \mathrm{~Hz}), 1.61$ (br, $1 \mathrm{H})$; ${ }^{13} \mathrm{C}$ NMR (125 MHz, $\mathrm{CDCl}_{3}$ ) 158.6, 142.9, 141.0, 132.6, 129.3, 128.5, 127.3, 127.2, 115.1, 113.8, 65.0, 55.3, 50.7; EI-MS [m/z] $253\left(\mathrm{M}^{+}\right)$; HPLC (Daicel CHIRALCEL OD-H; hexanes:2-propanol = 99.7:0.3, detection wavelength $=230 \mathrm{~nm}$, flow rate $=0.5 \mathrm{~mL} / \mathrm{min}$ ) $\mathrm{Tr}=24.3 \mathrm{~min}$ (major) and $31.5 \mathrm{~min}$ (minor).

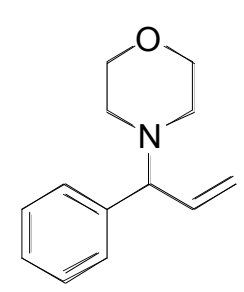

$N$-(1'-phenyl-2'-propenyl)morpholine (Table 2, entry 10): ${ }^{6}{ }^{1} \mathrm{H}$ NMR (400 MHz, $\left.\mathrm{CDCl}_{3}\right)$ 7.36-7.22 (m, 5H), 5.90 (ddd, 1H, $\left.J=17.8,9.4,8.3 \mathrm{~Hz}\right)$, $5.24(\mathrm{~d}, 1 \mathrm{H}, J=17.0 \mathrm{~Hz}), 5.10(\mathrm{~d}, 1 \mathrm{H}, J=10.0 \mathrm{~Hz}), 3.75-3.65(\mathrm{~m}, 4 \mathrm{H})$, $3.62(\mathrm{~d}, 1 \mathrm{H}, J=8.8 \mathrm{~Hz}), 2.55-2.45(\mathrm{~m}, 2 \mathrm{H}), 2.38-2.28(\mathrm{~m}, 2 \mathrm{H}),{ }^{13} \mathrm{C}$ NMR $\left(125 \mathrm{MHz}, \mathrm{CDCl}_{3}\right)$ 141.6, 139.7, 128.6, 128.0, 127.2, 116.6, 75.5, 67.1, 52.0; EI-MS [m/z] $203 \quad\left(\mathrm{M}^{+}\right) ; \quad$ HPLC (Daicel CHIRALCEL OJ-H; hexanes:2-propanol:diethylamine $=99.7: 0.3: 0.01$, detection wavelength $=210 \mathrm{~nm}$, flow rate $=0.5 \mathrm{~mL} / \mathrm{min}$ ) $\mathrm{Tr}=19.6 \mathrm{~min}$ (minor) and $24.6 \mathrm{~min}$ (major).

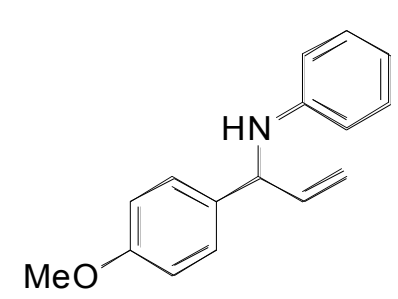

$N$-Phenyl-1-(p-methoxyphenyl)-2-propenylamine (Table 2, entry 11): ${ }^{5} \mathrm{H}$ NMR (400 $\left.\mathrm{MHz}, \mathrm{CDCl}_{3}\right)$ 7.31-7.26 (m, 2H), 7.16-7.11 (m, 2H), 6.90-6.85 (m, 2H), $6.69(\mathrm{t}, 1 \mathrm{H}, J=7.3 \mathrm{~Hz})$, $6.60(\mathrm{~d}, 2 \mathrm{H}, J=7.7 \mathrm{~Hz}), 6.02(\mathrm{ddd}, 1 \mathrm{H}, J=17.1,10.2,5.8 \mathrm{~Hz})$, $5.26(\mathrm{dt}, 1 \mathrm{H}, J=17.1,1.3 \mathrm{~Hz}), 5.21(\mathrm{dt}, 1 \mathrm{H}, J=10.2,1.2 \mathrm{~Hz})$, $4.88(\mathrm{~d}, 1 \mathrm{H}, J=5.8 \mathrm{~Hz}), 4.1(\mathrm{br}, 1 \mathrm{H}), 3.79(\mathrm{~s}, 3 \mathrm{H}) ;{ }^{13} \mathrm{C} \mathrm{NMR}\left(125 \mathrm{MHz}, \mathrm{CDCl}_{3}\right) 158.9$, 147.2, 139.2, 133.9, 129.1, 128.3, 117.6, 115.8, 114.1, 113.6, 60.2, 55.3; EI-MS [m/z] 239 $\left(\mathrm{M}^{+}\right)$; HPLC (Daicel CHIRALCEL OD-H; hexanes:2-propanol = 19:1, detection wavelength $=230 \mathrm{~nm}$, flow rate $=0.5 \mathrm{~mL} / \mathrm{min}$ ) $\mathrm{Tr}=14.7 \mathrm{~min}$ (major) and $16.3 \mathrm{~min}$ (minor). 


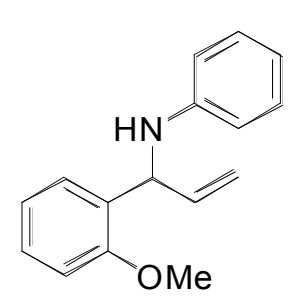

$\mathrm{N}$-Phenyl-1-(o-methoxyphenyl)-2-propenylamine (Table 2, entry 12): ${ }^{5} \mathrm{H}$ NMR (400 MHz, $\left.\mathrm{CDCl}_{3}\right) 7.31(\mathrm{dd}, 1 \mathrm{H}, J=7.4,1.6 \mathrm{~Hz}), 7.24$ (ddd, $1 \mathrm{H}, J=8.0,7.6,1.7 \mathrm{~Hz}), 7.15-7,08(\mathrm{~m}, 2 \mathrm{H}), 6.94-6.87(\mathrm{~m}, 2 \mathrm{H})$, $6.67(\mathrm{t}, 1 \mathrm{H}, J=7.3 \mathrm{~Hz}), 6.61(\mathrm{~d}, 2 \mathrm{H}, J=7.7 \mathrm{~Hz}), 6.07$ (ddd, $1 \mathrm{H}, J=17.2$, $10.2,5.4 \mathrm{~Hz}), 5.34(\mathrm{~d}, 1 \mathrm{H}, J=5.4 \mathrm{~Hz}), 5.21(\mathrm{dt}, 1 \mathrm{H}, J=17.2,1.4 \mathrm{~Hz})$, $5.16(\mathrm{dd}, 1 \mathrm{H}, J=10.2,1.4 \mathrm{~Hz}), 4.4(\mathrm{br}, 1 \mathrm{H}), 3.86(\mathrm{~s}, 3 \mathrm{H}) ;{ }^{13} \mathrm{C} \mathrm{NMR}\left(125 \mathrm{MHz}, \mathrm{CDCl}_{3}\right)$ 156.9, 147.3, 138.6, 129.7, 129.0, 128.4, 127.8, 120.8, 117.4, 115.1, 113.5, 110.8, 55.5, 54.7; EI-MS [m/z] $239\left(\mathrm{M}^{+}\right)$; HPLC (Daicel CHIRALCEL OD-H; hexanes:2-propanol = 99.95:0.05, detection wavelength $=230 \mathrm{~nm}$, flow rate $=1.0 \mathrm{~mL} / \mathrm{min}$ ) $\mathrm{Tr}=27.4 \mathrm{~min}$ (major) and $52.5 \mathrm{~min}$ (minor).

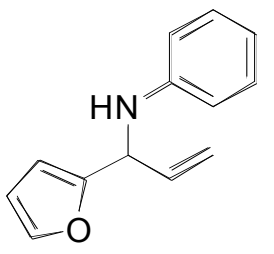

$\boldsymbol{N}$-Phenyl-1-(2-furyl)-2-propenylamine (Table 2, entry 13): ${ }^{1} \mathrm{H}$ NMR $\left(400 \mathrm{MHz}, \mathrm{CDCl}_{3}\right) 7.38(\mathrm{dd}, 1 \mathrm{H}, J=1.7 \mathrm{~Hz}, 0.7 \mathrm{~Hz}), 7.20-7.14(\mathrm{~m}, 2 \mathrm{H})$, $6.74(\mathrm{t}, 1 \mathrm{H}, J=7.4 \mathrm{~Hz}), 6.67(\mathrm{~d}, 2 \mathrm{H}, J=7.7 \mathrm{~Hz}), 6.33(\mathrm{dd}, 1 \mathrm{H}, J=3.2$, $1,8 \mathrm{~Hz}), 6.23(\mathrm{~d}, 1 \mathrm{H}, J=3.2 \mathrm{~Hz}), 6.05(\mathrm{ddd}, 1 \mathrm{H}, J=17.1,10.2,5.7 \mathrm{~Hz})$, $5.34(\mathrm{dt}, 1 \mathrm{H}, J=17.1,1.2 \mathrm{~Hz}), 5.28(\mathrm{dt}, 1 \mathrm{H}, J=10.2,1.2 \mathrm{~Hz}), 5.08(\mathrm{~d}, 1 \mathrm{H}, J=5.6 \mathrm{~Hz}), 4.3$ (br, 1H); ${ }^{13} \mathrm{C}$ NMR (125 MHz, $\left.\mathrm{CDCl}_{3}\right)$ 154.3, 146.8, 142.1, 136.3, 129.1, 118.1, 116.9, 113.7, 110.3, 106.7, 54.5; EI-MS [m/z] $199\left(\mathrm{M}^{+}\right)$; HPLC (Daicel CHIRALCEL OD-H; hexanes:2-propanol $=19: 1$, detection wavelength $=230 \mathrm{~nm}$, flow rate $=0.5 \mathrm{~mL} / \mathrm{min}) \operatorname{Tr}=$ $12.1 \mathrm{~min}$ (minor) and $14.3 \mathrm{~min}$ (major).

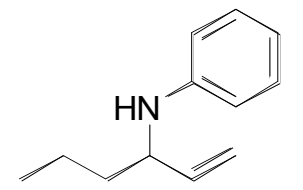

3-Phenylamino-1-hexene (Table 2, entry 14): ${ }^{1} \mathrm{H}$ NMR $(400 \mathrm{MHz}$, $\left.\mathrm{CDCl}_{3}\right)$ 7.18-7.12 (m, 2H), $6.68(\mathrm{t}, 1 \mathrm{H}, J=7.3 \mathrm{~Hz}), 6.61(\mathrm{~d}, 2 \mathrm{H}, J=7.8$ $\mathrm{Hz}), 5.73(\mathrm{ddd}, 1 \mathrm{H}, J=17.2,10.3,6.2 \mathrm{~Hz}), 5.20(\mathrm{dt}, 1 \mathrm{H}, J=17.2,1.3$ $\mathrm{Hz}), 5.11(\mathrm{dt}, 1 \mathrm{H}, J=10.3,1.2 \mathrm{~Hz}), 3.7(\mathrm{br}, 1 \mathrm{H}), 3.81(\mathrm{q}, 1 \mathrm{H}, J=6.4 \mathrm{~Hz}), 1.63-1.37(\mathrm{~m}$, 4H), $0.94(\mathrm{t}, 3 \mathrm{H}, J=7.3 \mathrm{~Hz}) ;{ }^{13} \mathrm{C}$ NMR $\left(125 \mathrm{MHz}, \mathrm{CDCl}_{3}\right)$ 147.7, 140.2, 129.1, 117.1, 114.9, 113.3, 55.7, 38.0, 19.1, 14.0; EI-MS [m/z] $175\left(\mathrm{M}^{+}\right)$; HPLC (Daicel CHIRALCEL OD-H; hexanes:2-propanol $=99.95: 0.05$, detection wavelength $=230 \mathrm{~nm}$, flow rate $=0.5$ $\mathrm{mL} / \mathrm{min}$ ) $\mathrm{Tr}=30.0 \mathrm{~min}$ (major) and $38.1 \mathrm{~min}$ (minor). 


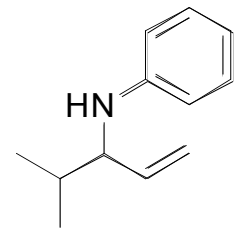

4-Methyl-3-phenylamino-1-pentene (Table 2, entry 15) ${ }^{5}$ : ${ }^{1} \mathrm{H}$ NMR (400 $\left.\mathrm{MHz}, \mathrm{CDCl}_{3}\right) 7.18-7.12(\mathrm{~m}, 2 \mathrm{H}), 6.66(\mathrm{t}, 1 \mathrm{H}, J=7.3 \mathrm{~Hz}), 6.60(\mathrm{~d}, 2 \mathrm{H}, J=$ $8.0 \mathrm{~Hz}$ ), 5.72 (ddd, $1 \mathrm{H}, J=17.2,10.3,6.3 \mathrm{~Hz}), 5.19(\mathrm{~d}, 1 \mathrm{H}, J=17.1 \mathrm{~Hz})$, $5.16(\mathrm{~d}, 1 \mathrm{H}, J=10.2 \mathrm{~Hz}), 3.71(\mathrm{br}, 1 \mathrm{H}), 3.65(\mathrm{t}, 1 \mathrm{H}, J=5.9 \mathrm{~Hz}), 1.87(\mathrm{qqd}$, $1 \mathrm{H}, J=6.8,6.8,5.7 \mathrm{~Hz}) .0 .99(\mathrm{~d}, 3 \mathrm{H}, J=6.8 \mathrm{~Hz}), 0.96(\mathrm{~d}, 3 \mathrm{H}, J=6.8 \mathrm{~Hz}) ;{ }^{13} \mathrm{C} \mathrm{NMR}(125$ $\left.\mathrm{MHz}, \mathrm{CDCl}_{3}\right)$ 147.9, 137.9, 129.1, 117.1, 116.0, 113.4, 61.5, 32.5, 18.8, 18.5; EI-MS [m/z] $175\left(\mathrm{M}^{+}\right)$; HPLC (Daicel CHIRALCEL OD-H; hexanes:2-propanol = 99.95:0.05, detection wavelength $=230 \mathrm{~nm}$, flow rate $=0.5 \mathrm{~mL} / \mathrm{min}$ ) $\mathrm{Tr}=22.0 \mathrm{~min}$ (major) and $24.0 \mathrm{~min}$ (minor).

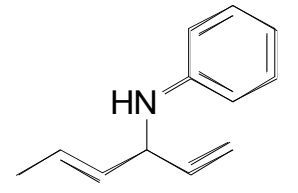

3-Phenylamino-1,4-hexadiene (Table 2, entry 16) ${ }^{5}$ : ${ }^{1} \mathrm{H}$ NMR (400 $\left.\mathrm{MHz}, \mathrm{CDCl}_{3}\right) 7.19-7.13(\mathrm{~m}, 2 \mathrm{H}), 6.70(\mathrm{t}, 1 \mathrm{H}, J=7.3 \mathrm{~Hz}), 6.63(\mathrm{~d}, 2 \mathrm{H}, J$ $=7.7 \mathrm{~Hz}), 5.86(\mathrm{ddd}, 1 \mathrm{H}, J=17.2,10.3,5.7 \mathrm{~Hz}), 5.71(\mathrm{dqd}, 1 \mathrm{H}, J=15.4$, 6.4, $1.1 \mathrm{~Hz}), 5.49(\mathrm{ddq}, 1 \mathrm{H}, J=15.3,6.2,1.5 \mathrm{~Hz}), 5.25(\mathrm{dt}, 1 \mathrm{H}, J=17.2,1.4 \mathrm{~Hz}), 5.16(\mathrm{dt}$, $1 \mathrm{H}, J=10.3,1.3 \mathrm{~Hz}$ ), 4.36 (t, 1H, $J=6.0 \mathrm{~Hz}$ ), 3.93 (br, $1 \mathrm{H}), 1.71$ (dt, $3 \mathrm{H}, J=6.4,1.2 \mathrm{~Hz}$ ); ${ }^{13} \mathrm{C}$ NMR (125 MHz, $\left.\mathrm{CDCl}_{3}\right)$ 147.2, 138.8, 131.0, 129.0, 127.3, 117.4, 115.3, 113.6, 57.6, 17.8; EI-MS [m/z] $173\left(\mathrm{M}^{+}\right)$; HPLC (Daicel CHIRALCEL OD-H; hexanes:2-propanol = 99.95:0.05, detection wavelength $=230 \mathrm{~nm}$, flow rate $=0.5 \mathrm{~mL} / \mathrm{min}$ ) $\mathrm{Tr}=32.9 \mathrm{~min}$ (major) and $36.1 \mathrm{~min}$ (minor).

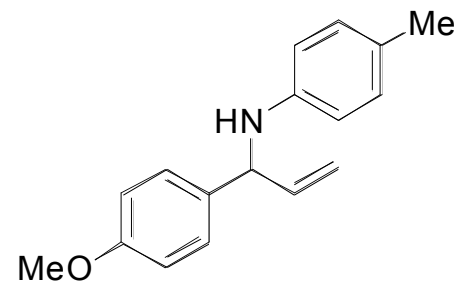

$N$-(1-(4-methoxyphenyl)allyl)-4-methylbenzenamine (Table 3, entry 5): ${ }^{1} \mathrm{H}$ NMR (400 MHz, $\left.\mathrm{CDCl}_{3}\right) 7.33(\mathrm{~d}, 2 \mathrm{H}, J$ $=8.8 \mathrm{~Hz}), 6.99(\mathrm{~d}, 1 \mathrm{H}, J=5.2 \mathrm{~Hz}), 6.92(\mathrm{dt}, 2 \mathrm{H}, J=6.8,1.6$ $\mathrm{Hz}), 6.57(\mathrm{~d}, 2 \mathrm{H}, J=8.4 \mathrm{~Hz}), 6.05(\mathrm{ddd}, 1 \mathrm{H}, J=16.8,10.0$, $5.6 \mathrm{~Hz}), 5.30(\mathrm{dd}, 1 \mathrm{H}, J=16.8,1.2 \mathrm{~Hz}), 5.24(\mathrm{dd}, 1 \mathrm{H}, J=10.0$, $1.2 \mathrm{~Hz}$ ), 3.92 (br s, 1H), 3.83 (s, 3H), 2.26 (s, 3H); ${ }^{13} \mathrm{C} \mathrm{NMR}\left(125 \mathrm{MHz}, \mathrm{CDCl}_{3}\right.$ ) 158.7, 144.9, 139.1, 134.0, 129.5, 128.2, 126.6, 115.5, 113.9, 113.6, 60.4, 55.2, 20.3; EI-MS [m/z] $253\left(\mathrm{M}^{+}\right) ;[\alpha]_{\mathrm{D}}^{20}=+20.0\left(\mathrm{c} 1.5, \mathrm{CHCl}_{3}\right) ;$ HPLC (Daicel CHIRALCEL OD-H; hexanes:2-propanol $=19: 1$, detection wavelength $=230 \mathrm{~nm}$, flow rate $=1.0 \mathrm{~mL} / \mathrm{min}) \operatorname{Tr}=$ 
25.1 min (major) and 37.2 min (major); Anal. Calcd for C 80.60, H 7.56, N 5.53; Found: C 80.22, H 7.71, N 5.64.

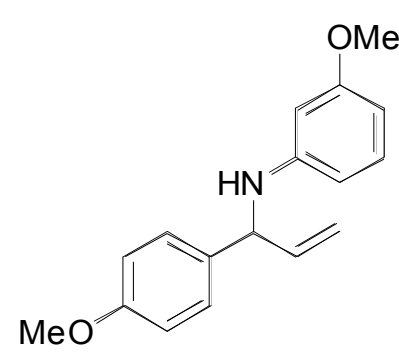

3-methoxy- $\mathrm{N}$-(1-(4-methoxyphenyl)allyl)benzenamine (Table 3, entry 6) : ${ }^{1} \mathrm{H}$ NMR (400 MHz, $\left.\mathrm{CDCl}_{3}\right) 7.28(\mathrm{~d}, 2 \mathrm{H}, J=6.8 \mathrm{~Hz})$, $7.04(\mathrm{t}, 1 \mathrm{H}, J=8.0 \mathrm{~Hz}), 6.88(\mathrm{~d}, 2 \mathrm{H}, J=8.0 \mathrm{~Hz}), 6.26(\mathrm{dd}, 1 \mathrm{H}, J$ $=8.4,2.4 \mathrm{~Hz}), \quad 6.22(\mathrm{dd}, 1 \mathrm{H}, J=8.0,2.0 \mathrm{~Hz}), 6.15(\mathrm{t}, 1 \mathrm{H}, J=$ $2.4 \mathrm{~Hz}), 6.03(\mathrm{ddd}, 1 \mathrm{H}, J=16.0,10.0,5.6 \mathrm{~Hz}), 5.26(\mathrm{dt}, 1 \mathrm{H}, J=$ $17.2,1.2 \mathrm{~Hz}$ ), $5.20(\mathrm{dt}, 1 \mathrm{H}, J=10.4,1.6 \mathrm{~Hz}), 4.86$ (br s, $1 \mathrm{H})$, 4.01 (br s, 1H), 3.79 (s, 3H), 3.77 (s, 3H); ${ }^{13} \mathrm{C}$ NMR (125 MHz, $\left.\mathrm{CDCl}_{3}\right)$ 160.5, 158.8, 148.6, 139.0, 133.8, 129.7, 128.2, 115.8, 114.0, 106.6, 102.5, 99.4, 60.1, 55.2, 55.0; EI-MS [m/z] $269\left(\mathrm{M}^{+}\right) ;[\alpha]_{\mathrm{D}}^{20}=+13.5$ (c 3.20, $\mathrm{CHCl}_{3}$ ); HPLC (Daicel CHIRALCEL OD-H; hexanes:2-propanol $=19: 1$, detection wavelength $=230 \mathrm{~nm}$, flow rate $=1.0 \mathrm{~mL} / \mathrm{min}) \operatorname{Tr}=$ 49.5 min (major) and 61.5 min (minor); Anal. Calcd for C 75.81, H 7.11, N 5.20; Found C 75.32, H 7.24, N 5.20.

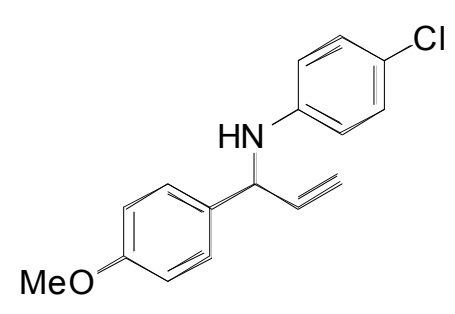

4-chloro- $\mathrm{N}$-(1-(4-methoxyphenyl)allyl)benzenamine (Table 3, entry 7) : ${ }^{1} \mathrm{H} \mathrm{NMR}\left(400 \mathrm{MHz}, \mathrm{CDCl}_{3}\right) 7.26$ (dt, $2 \mathrm{H}, J=8.4$, $2.0 \mathrm{~Hz}), 7.07(\mathrm{dt}, 2 \mathrm{H}, J=9.2,2.4 \mathrm{~Hz}), 6.88(\mathrm{dt}, 2 \mathrm{H}, J=8.8$, $2.0 \mathrm{~Hz}$ ), $6.51(\mathrm{dt}, 2 \mathrm{H}, J=8.8,2.0 \mathrm{~Hz}), 5.99$ (ddd, $1 \mathrm{H}, J=16.0$, $10.0,5.6 \mathrm{~Hz}), 5.25(\mathrm{dt}, 1 \mathrm{H}, J=13.2,1.6 \mathrm{~Hz}), 5.22(\mathrm{ddd}, 1 \mathrm{H}, J$ $=5.2,1.2 \mathrm{~Hz}), 4.83(\mathrm{~d}, 1 \mathrm{H}, J=5.2 \mathrm{~Hz}), 4.00(\mathrm{br} \mathrm{s}, 1 \mathrm{H}), 3.80(\mathrm{~s}$, $3 \mathrm{H}) ;{ }^{13} \mathrm{C}$ NMR $\left(125 \mathrm{MHz}, \mathrm{CDCl}_{3}\right) 160.0,145.7,138.7,133.4,128.8,128.2,122.0,115.9$, 114.5, 114.1, 60.0, 55.2; EI-MS [m/z] $273\left(\mathrm{M}^{+}\right) ;[\alpha]_{\mathrm{D}}{ }^{20}=15.5\left(\mathrm{c} 1.70, \mathrm{CHCl}_{3}\right) ; \mathrm{HPLC}$ (Daicel CHIRALCEL OD-H; hexanes:2-propanol $=19: 1$, detection wavelength $=230 \mathrm{~nm}$, flow rate $=1.0 \mathrm{~mL} / \mathrm{min}$ ) $\mathrm{Tr}=31.4 \mathrm{~min}$ (major) and $44.0 \mathrm{~min}$ (minor). 


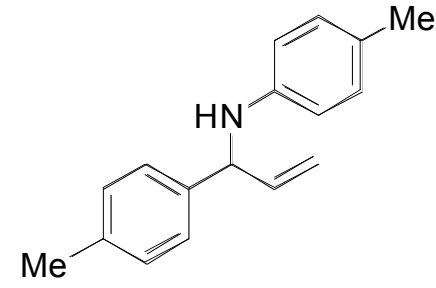

4-methyl- $N$-(1-p-tolylallyl)benzenamine (Table 3, entry 8): ${ }^{1} \mathrm{H}$ NMR (400 MHz, $\left.\mathrm{CDCl}_{3}\right) 7.26(\mathrm{~d}, 2 \mathrm{H}, J=8.0 \mathrm{~Hz}), 7.15$ (d, $2 \mathrm{H}, J=8.0 \mathrm{~Hz}), 6.95(\mathrm{~d}, 2 \mathrm{H}, J=8.0 \mathrm{~Hz}), 6.52(\mathrm{~d}, 2 \mathrm{H}, J=8.0$ $\mathrm{Hz}$ ), 6.02 (ddd, 1H, $J=16.0,10.0,8.0 \mathrm{~Hz}), 5.27$ (dt, 1H, $J=$ $16.8,1.6 \mathrm{~Hz}), 5.20(\mathrm{dt}, 1 \mathrm{H}, J=10.4,1.6 \mathrm{~Hz}), 4.87$ (d, 1H, $J=$ $4.0 \mathrm{~Hz}), 2.34$ (s, 3H), 2.22 (s, 3H); ${ }^{13} \mathrm{C} \mathrm{NMR}\left(125 \mathrm{MHz}, \mathrm{CDCl}_{3}\right)$ 144.9, 139.3, 139.0, 137.0, $129.5,129.4,127.0,126.6,115.6,113.6,60.8,21.1,20.3$; EI-MS [m/z] $237\left(\mathrm{M}^{+}\right) ;[\alpha]_{\mathrm{D}}{ }^{20}=$ 17.1 (c 1.80, $\mathrm{CHCl}_{3}$ );HPLC (Daicel CHIRALCEL OD-H; hexanes:2-propanol = 19:1, detection wavelength $=230 \mathrm{~nm}$, flow rate $=1.0 \mathrm{~mL} / \mathrm{min}$ ) $\mathrm{Tr}=24.5 \mathrm{~min}$ (major) and 32.8 min (minor); Anal. Calcd for C 86.03, H 8.07, N 5.90; Found: C 85.66, H 8.31, N 5.97.

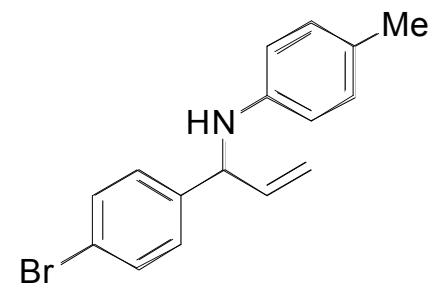

N-(1-(4-bromophenyl)allyl)-4-methylbenzenamine (Table 3, entry 9): ${ }^{1} \mathrm{H}$ NMR $\left(400 \mathrm{MHz}, \mathrm{CDCl}_{3}\right) 7.46(\mathrm{dt}, 2 \mathrm{H}, J=8.0,2.0$ $\mathrm{Hz}), 7.26(\mathrm{dt}, 2 \mathrm{H}, J=8.4,2.0 \mathrm{~Hz}), 6.94(\mathrm{~d}, 2 \mathrm{H}, J=8.0 \mathrm{~Hz})$, $6.48(\mathrm{~d}, 2 \mathrm{H}, J=8.0 \mathrm{~Hz}), 5.99(\mathrm{ddd}, 1 \mathrm{H}, J=17.2,10.0,6.0 \mathrm{~Hz})$, $5.24(\mathrm{dt}, 1 \mathrm{H}, J=10.0,1.6 \mathrm{~Hz}), 5.21(\mathrm{dt}, 1 \mathrm{H}, J=1.6,1.6 \mathrm{~Hz})$, $4.86(\mathrm{~d}, 1 \mathrm{H}, J=4.0 \mathrm{~Hz}), 3.90(\mathrm{br} \mathrm{s}, 1 \mathrm{H}), 2.21$ (s, 3H); ${ }^{13} \mathrm{C} \mathrm{NMR}\left(125 \mathrm{MHz}, \mathrm{CDCl}_{3}\right) 144.5$, 141.0, 138.7, 131.7, 129.6, 128.8, 127.0, 121.1, 116.5, 113.6, 60.6, 20.3; EI-MS [m/z] 301 $\left(\mathrm{M}^{+}\right) ;[\alpha]_{\mathrm{D}}{ }^{20}=63.0\left(\mathrm{c} 2.0, \mathrm{CHCl}_{3}\right)$; HPLC (Daicel CHIRALCEL OD-H; hexanes:2-propanol $=19: 1$, detection wavelength $=230 \mathrm{~nm}$, flow rate $=1.0 \mathrm{~mL} / \mathrm{min}$ ) $\mathrm{Tr}=37.1 \mathrm{~min}$ (major) and 55.1 min (minor); Anal. Calcd for C 63.59, H 5.34, N 4.63; Found: C 63.20, H 5.37, N 4.59 . 
Table $\mathbf{S} 1^{\mathrm{a}}$ Asymmetric allylic substitutions of $\mathbf{1 a}$ with $\mathbf{2 a}$ in the presence of titanium alkoxides

\begin{tabular}{|c|c|c|c|c|c|c|}
\hline \multirow[b]{2}{*}{ Entry } & \multirow{2}{*}{$\begin{array}{c}1 \mathbf{a} \\
(1.0 \text { eq. }) \\
\begin{array}{c}\text { Titanium alkoxide } \\
\text { (eq.) }\end{array}\end{array}$} & \multirow{2}{*}{$\begin{array}{c}\begin{array}{c}\mathrm{PhNH}_{2} \\
\text { 2a } \\
(1.2 \text { eq. })\end{array} \\
\text { Solvent }\end{array}$} & \multicolumn{2}{|c|}{$\begin{array}{c}\text { Chiral Ir catalyst (5a) } \\
(2 \mathrm{~mol} \%)\end{array}$} & \multirow{2}{*}{$\frac{3 a a}{3 a a / 4 a a^{b}}$} & \multirow[b]{2}{*}{ ee of $3 a a(\%)$} \\
\hline & & & Additive & $\begin{array}{c}\text { Isolated yield } \\
\text { of } \mathbf{3 a a}(\%)\end{array}$ & & \\
\hline 1 & $\mathrm{Ti}\left(\mathrm{O}^{i} \mathrm{Pr}\right)_{4}(0.5)$ & THF & $4 \AA ̊ M S$ & 30 & $92 / 8$ & 82 \\
\hline $2^{c}$ & $\mathrm{Ti}\left(\mathrm{O}^{j} \mathrm{Pr}\right)_{4}(0.5)$ & THF & $4 \AA \mathrm{MS}$ & 41 & $76 / 24$ & 57 \\
\hline 3 & $\mathrm{Ti}\left(\mathrm{O}^{i} \mathrm{Pr}\right)_{4}(1.2)$ & THF & $3 \AA ̊ M S$ & 20 & $91 / 9$ & 80 \\
\hline 4 & $\mathrm{Ti}\left(\mathrm{O}^{i} \mathrm{Pr}\right)_{4}(1.2)$ & THF & $4 \AA ̊ M S$ & 16 & $89 / 11$ & 81 \\
\hline 5 & $\mathrm{Ti}\left(\mathrm{O}^{j} \mathrm{Pr}\right)_{4}(1.2)$ & THF & $5 \AA ̊ M S$ & 7 & $60 / 40$ & 74 \\
\hline 6 & $\mathrm{Ti}(\mathrm{OEt})_{4}(1.2)$ & THF & $4 \AA ̊ \mathrm{MS}$ & 16 & $94 / 6$ & 91 \\
\hline 7 & $\mathrm{Ti}\left(\mathrm{O}^{t} \mathrm{Bu}\right)_{4}(0.5)$ & THF & $4 \AA ̊ \mathrm{MS}$ & 36 & $92 / 8$ & 80 \\
\hline 8 & $\mathrm{Ti}\left(\mathrm{O}^{t} \mathrm{Bu}\right)_{4}(1.2)$ & THF & $4 \AA ̊ M S$ & 30 & $94 / 6$ & 89 \\
\hline $9^{d}$ & $\mathrm{Ti}\left(\mathrm{O}^{t} \mathrm{Bu}\right)_{4}(1.2)$ & THF & $4 \AA ̊ M S$ & 28 & $93 / 7$ & 85 \\
\hline $10^{e}$ & $\mathrm{Ti}\left(\mathrm{O}^{t} \mathrm{Bu}\right)_{4}(1.2)$ & THF & $4 \AA ̊ M S$ & 30 & $95 / 5$ & 88 \\
\hline 11 & $\mathrm{Ti}\left(\mathrm{O}^{t} \mathrm{Bu}\right)_{4}(3.0)$ & THF & $4 \AA \mathrm{MS}$ & 21 & $94 / 6$ & 91 \\
\hline $12^{f}$ & $\mathrm{Ti}\left(\mathrm{O}^{t} \mathrm{Bu}\right)_{4}(1.2)$ & THF & $4 \AA \mathrm{MS}$ & 35 & $92 / 8$ & 85 \\
\hline 13 & $\mathrm{Ti}\left(\mathrm{O}^{t} \mathrm{Bu}\right)_{4}(1.2)$ & THF & $3 \AA ̊ M S$ & 25 & $92 / 8$ & 89 \\
\hline $14^{f}$ & $\mathrm{Ti}\left(\mathrm{O}^{t} \mathrm{Bu}\right)_{4}(1.2)$ & THF & 3Å MS & 33 & $93 / 7$ & 87 \\
\hline 15 & $\mathrm{Ti}\left(\mathrm{O}^{t} \mathrm{Bu}\right)_{4}(0.5)$ & THF/DMF (4/1) & $4 \AA ̊ M S$ & 32 & $94 / 6$ & 75 \\
\hline 16 & $\mathrm{Ti}\left(\mathrm{O}^{t} \mathrm{Bu}\right)_{4}(1.2)$ & THF/DMF (4/1) & $4 \AA ̊ M S$ & 26 & $92 / 8$ & 84 \\
\hline 17 & $\mathrm{Ti}\left(\mathrm{O}^{t} \mathrm{Bu}\right)_{4}(1.2)$ & $\mathrm{CH}_{3} \mathrm{CN}$ & $4 \AA ̊ M S$ & 34 & $93 / 7$ & 44 \\
\hline 18 & $\mathrm{Ti}\left(\mathrm{O}^{t} \mathrm{Bu}\right)_{4}(1.2)$ & DMF & $4 \AA \mathrm{MS}$ & 32 & $95 / 5$ & 77 \\
\hline 19 & $\mathrm{Ti}\left(\mathrm{O}^{t} \mathrm{Bu}\right)_{4}(1.2)$ & $\mathrm{CH}_{3} \mathrm{NO}_{2}$ & $4 \AA ̊ M S$ & trace & - & - \\
\hline 20 & $\mathrm{Ti}\left(\mathrm{O}^{t} \mathrm{Bu}\right)_{4}(1.2)$ & diglyme & $4 \AA \mathrm{MS}$ & 35 & $93 / 7$ & 90 \\
\hline $21^{\mathrm{g}}$ & $\mathrm{Ti}\left(\mathrm{O}^{t} \mathrm{Bu}\right)_{4}(1.2)$ & diglyme & $4 \AA ̊ \mathrm{MS}$ & n.p. & $\sim^{h}$ & - \\
\hline 22 & $\mathrm{Ti}\left(\mathrm{O}^{t} \mathrm{Bu}\right)_{4}(1.2)$ & toluene & $4 \AA ̊ \mathrm{MS}$ & 32 & $90 / 10$ & 87 \\
\hline $23^{g}$ & $\mathrm{Ti}\left(\mathrm{O}^{t} \mathrm{Bu}\right)_{4}(1.2)$ & toluene & $4 \AA \mathrm{MS}$ & trace & $\Psi^{h}$ & - \\
\hline 24 & $\mathrm{Ti}\left(\mathrm{O}^{t} \mathrm{Bu}\right)_{4}(1.2)$ & DME & $4 \AA \mathrm{MS}$ & 37 & $94 / 6$ & 88 \\
\hline $25^{\mathrm{i}}$ & $\mathrm{Ti}\left(\mathrm{O}^{t} \mathrm{Bu}\right)_{4}(1.2)$ & DME & $4 \AA ̊ M S$ & $<44$ & $65 / 35$ & 62 \\
\hline
\end{tabular}

a The reaction was performed with $1 \mathrm{a}(1.0 \mathrm{mmol})$ and $2 \mathrm{2a}(1.2 \mathrm{mmol})$ in THF $(0.5 \mathrm{~mL})$ at $50{ }^{\circ} \mathrm{C}$ for $24 \mathrm{~h}$ in the presence of the Ir complex $(2 \mathrm{~mol} \%)$ prepared from $[\operatorname{Ir}(\mathrm{COD}) \mathrm{Cl}]_{2}(0.010 \mathrm{mmol})$ and $5 \mathbf{a}(0.020 \mathrm{mmol})$, titanium alkoxide and powdered $4 \AA$ molecular sieves $(50 \mathrm{mg})$ unless otherwise noted. ${ }^{\mathrm{b}}$ Ratio of 3 aa and 4aa determined by ${ }^{1} \mathrm{H}$ NMR analysis of the crude reaction mixture. ${ }^{\mathrm{c}}$ For $48 \mathrm{~h}$. ${ }^{\mathrm{d}} 4 \AA \mathrm{A} \mathrm{MS}$ (100 mg) was used. ${ }^{\mathrm{e}} 3.0 \mathrm{mmol}$ of $2 \mathrm{a}$ was used. ${ }^{\mathrm{f}}$ The Ir catalyst $(4 \mathrm{~mol} \%)$ was used. ${ }^{\mathrm{g}}$ At $110^{\circ} \mathrm{C} .{ }^{\mathrm{h}}$ Linear products ( $N$-mono- and $\mathrm{N}, \mathrm{N}$-dicinnamylaniline) were obtained. i At $80^{\circ} \mathrm{C}$. n.p.: No desired product was obtained.

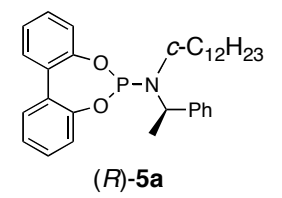


Table S2 ${ }^{\mathrm{a}}$ Screening of Lewis acid activator

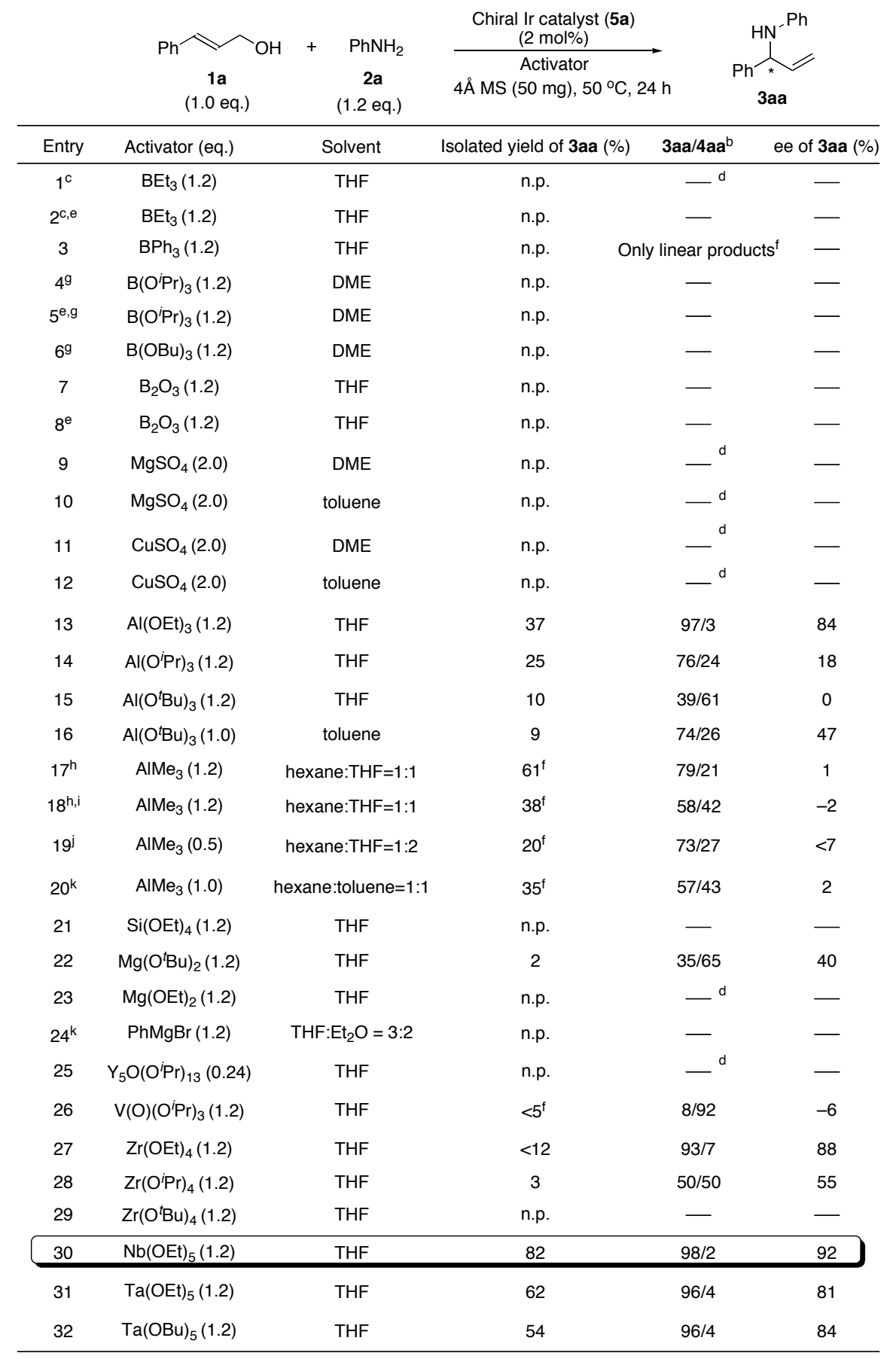

a The reaction was performed with $1 \mathrm{a}(1.0 \mathrm{mmol})$ and $\mathbf{2 a}(1.2 \mathrm{mmol})$ in THF $(0.5 \mathrm{~mL})$ at $50^{\circ} \mathrm{C}$ for $24 \mathrm{~h}$ in the presence of the Ir complex (2 mol\%) prepared from $[\operatorname{Ir}(C O D) C l]_{2}(0.010 \mathrm{mmol})$ and $5 \mathrm{a}(0.020 \mathrm{mmol})$, Lewis acid activator and powdered $4 \AA \AA$ molecular sieves $(50 \mathrm{mg})$ unless otherwise noted. ${ }^{\mathrm{b}}$ Ratio of $3 \mathbf{a a}$ and $4 \mathbf{a a}$ determined by ${ }^{1} \mathrm{H}$ NMR analysis of the crude reaction mixture. ${ }^{c}$ The amount of solvent was $1.4 \mathrm{~mL} .{ }^{\mathrm{d}} \mathrm{A}$ small amount of linear product (4aa) was obtained. e Without $4 \AA$ MS. ${ }^{\dagger} \mathrm{N}, \mathrm{N}$-dicinnamylaniline was also obtained. ${ }^{\mathrm{g}}$ At $80^{\circ} \mathrm{C}$. ${ }^{\mathrm{h}}$ The amount of solvent was $1.2 \mathrm{~mL}$. ${ }^{\mathrm{i}}$ At $\mathrm{rt}$ for $72 \mathrm{~h}$. ${ }^{\mathrm{j}}$ The amount of solvent was 0.75 $\mathrm{mL}$. ${ }^{\mathrm{k}}$ The amount of solvent was $1.0 \mathrm{~mL}$. n.p.: No desired product was obtaind. 
Table S3. Screening of conditions for the allylations with $\mathrm{Ti}\left(\mathrm{O}^{i} \mathrm{Pr}\right)_{4}, \mathrm{Nb}(\mathrm{OEt})_{5}$ and $\mathrm{Ta}(\mathrm{OEt})_{5}$ as activator

\begin{tabular}{llllll}
\hline Entry & Activator (eq.) & Catalyst & ${\text { Yield }(\%)^{\mathrm{b}}}^{\mathbf{3 a a} / \mathbf{4} \mathbf{a a}^{\mathrm{c}}}$ & $\mathrm{Ee}(\%)$ \\
\hline 1 & $\mathrm{Ti}\left(\mathrm{O}^{i} \mathrm{Pr}\right)_{4}(1.2)$ & $\mathrm{Ir} / \mathbf{5 a}$ & 16 & $89 / 11$ & 81 \\
2 & $\mathrm{Ti}\left(\mathrm{O}^{\prime} \mathrm{Bu}\right)_{4}(1.2)$ & $\mathrm{Ir} / \mathbf{5 a}$ & 30 & $94 / 6$ & 89 \\
3 & $\mathrm{Ta}(\mathrm{OEt})_{5}(1.2)$ & $\mathrm{Ir} / \mathbf{5 a}$ & 62 & $96 / 4$ & 81 \\
4 & $\mathrm{Nb}(\mathrm{OEt})_{5}(1.2)$ & $\mathrm{Ir} / \mathbf{5 a}$ & 82 & $98 / 2$ & 92 \\
$5^{\mathrm{d}}$ & $\mathrm{Nb}(\mathrm{OEt})_{5}(1.2)$ & $\mathrm{Ir} / \mathbf{5 a}$ & 47 & $91 / 9$ & 92 \\
6 & $\mathrm{Nb}(\mathrm{OEt})_{5}(0.5)$ & $\mathrm{Ir} / \mathbf{5 a}$ & 64 & $84 / 16$ & 63 \\
$7^{\mathrm{e}}$ & $\mathrm{Nb}(\mathrm{OEt})_{5}(1.2)$ & $\mathrm{Ir} / \mathbf{5 a}$ & 85 & $97 / 3$ & 92 \\
$8^{\mathrm{f}}$ & $\mathrm{Nb}(\mathrm{OEt})_{5}(1.2)$ & $\mathrm{Ir} / \mathbf{5 b}$ & 64 & $98 / 2$ & 94 \\
$9^{\mathrm{e}, \mathrm{g}}$ & $\mathrm{Nb}(\mathrm{OEt})_{5}(1.2)$ & $\mathrm{Ir} / \mathbf{5 a}$ & 42 & $98 / 2$ & 95 \\
\hline
\end{tabular}

${ }^{\mathrm{a}}$ The reaction was performed with $\mathbf{1 a}(1.0 \mathrm{mmol})$ and $\mathbf{2 a}(1.2 \mathrm{mmol})$ in $\mathrm{THF}(0.5 \mathrm{~mL})$ at $50{ }^{\circ} \mathrm{C}$ for $24 \mathrm{~h}$ in the presence of the $\mathrm{Ir}$ complex $(2 \mathrm{~mol} \%)$ prepared from $[\operatorname{Ir}(\mathrm{COD}) \mathrm{Cl}]_{2}(0.010 \mathrm{mmol})$ and $\mathbf{5 a}$ or $\mathbf{5 b}(0.020$ mmol), Lewis acid activator and powdered $4 \AA$ molecular sieves ( $4 \AA \mathrm{MS}, 50 \mathrm{mg}$ ) unless otherwise noted. ${ }^{\mathrm{b}}$ Isolated yield of branched product 3aa. ${ }^{\mathrm{c}}$ Ratio of 3aa and 4aa determined by ${ }^{1} \mathrm{H}$ NMR analysis of the crude reaction mixture. ${ }^{\mathrm{d}}$ Without $4 \AA$ MS. ${ }^{\mathrm{e}} 1.5 \mathrm{mmol}$ of $\mathbf{2 a}$ was used. ${ }^{\mathrm{f}}$ Ligand $\mathbf{5} \mathbf{b}$ was used. ${ }^{\mathrm{g}}$ The reaction was conducted at room temperature. 


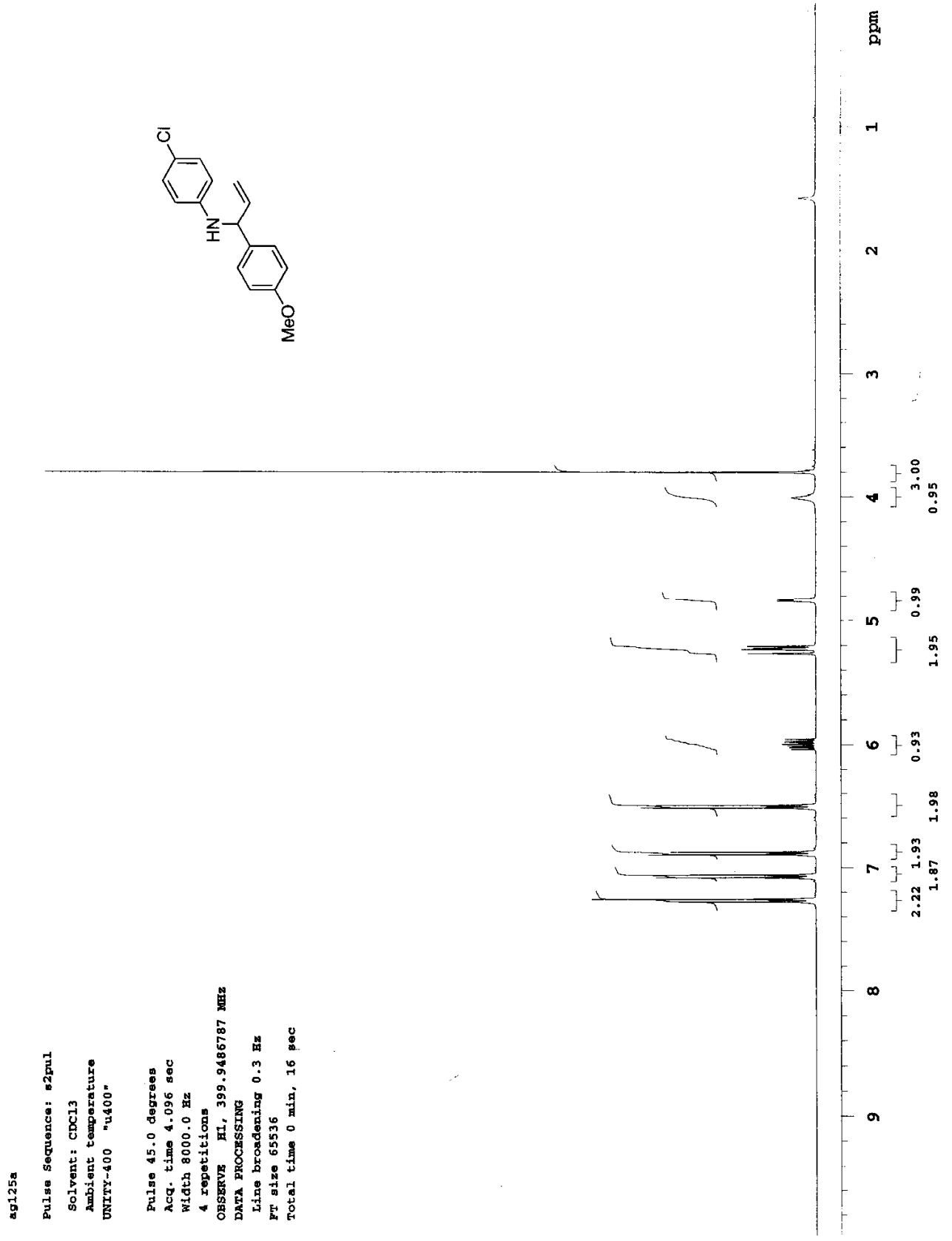

Figure S1. ${ }^{1} \mathrm{H}$ NMR of 4-chloro- $N$-(1-(4-methoxyphenyl)allyl)benzenamine. 

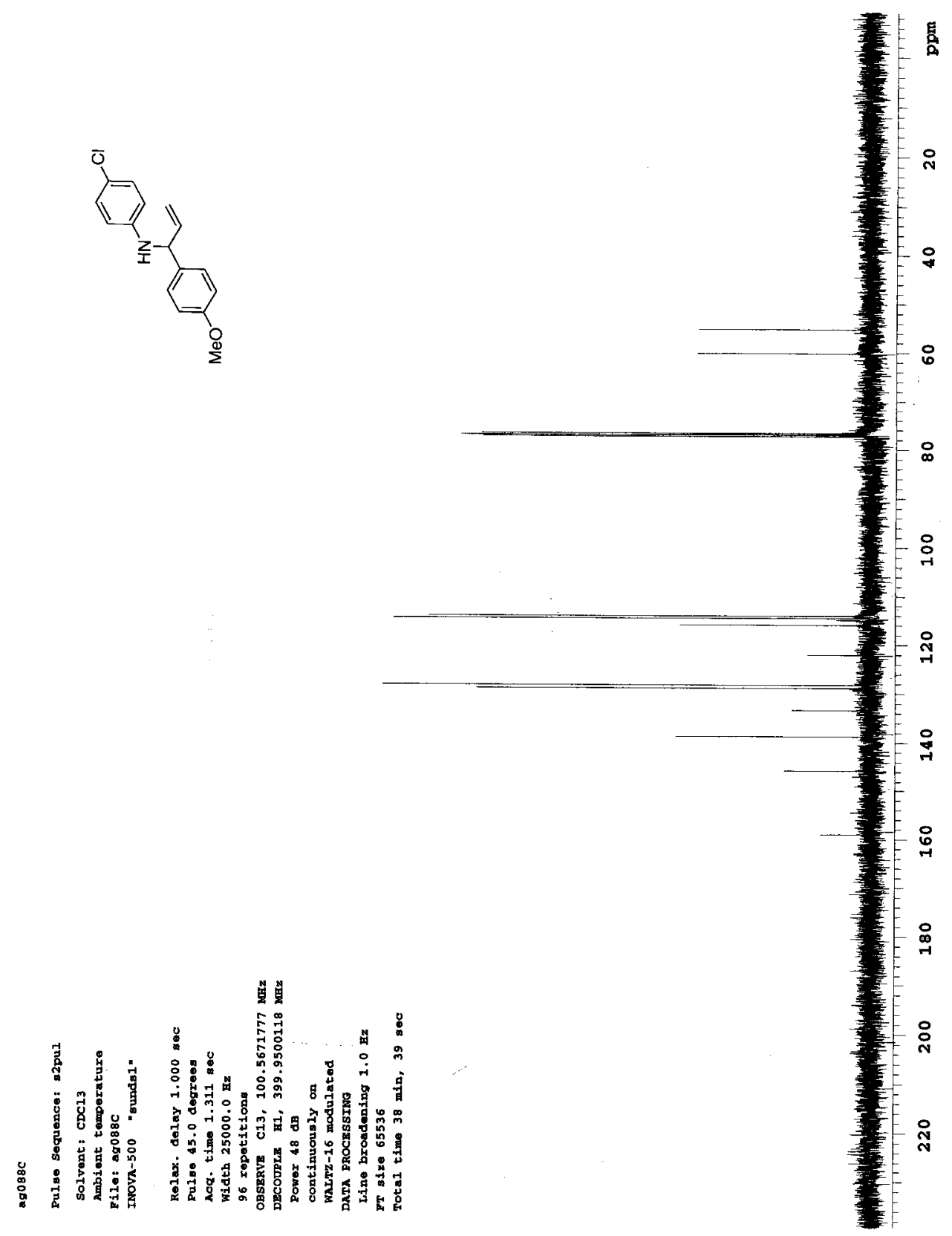

Figure S2. ${ }^{13} \mathrm{C}$ NMR of 4-chloro- $N$-(1-(4-methoxyphenyl)allyl)benzenamine. 


\section{References}

(1) Herde, J. L.; Lambert, J. C.; Senoff, C. V. Inorg. Synth. 1974, 15, 18.

(2) Leitner, A.; Shekhar, S.; Pouy, M. J.; Hartwig, J. F. J. Am. Chem. Soc. 2005, 127, 15506.

(3) Alexakis, A.; Rosset, S.; Allamand, J.; March, S.; Guillen, F.; Benhaim, C. Synlett 2001, 1375.

(4) Wu, Z. X.; Minhas, G. S.; Wen, D. Y.; Jiang, H. L.; Chen, K. X.; Zimniak, P.; Zheng, J. J. Med. Chem. 2004, 47, 3282.

(5) Shu, C. T.; Leitner, A.; Hartwig, J. F. Angew. Chem., Int. Ed. 2004, 43, 4797.

(6) Ohmura, T.; Hartwig, J. F. J. Am. Chem. Soc. 2002, 124, 15164. 